\title{
Co-infection by Cryptococcus neoformans fungaemia and Non tuberculous Mycobacterium together with Pneumocystis jiroveci pneumonia in a patient with newly diagnosed HIV infection
}

\author{
Eihab Subahi ${ }^{1}$, safwan aljafar ${ }^{1}$, haidar barjas ${ }^{1}$, Mohamed Abdelrazek $^{1}$, and Fatima Rasoul ${ }^{1}$ \\ ${ }^{1}$ Hamad Medical Corporation
}

February 24, 2021

\begin{abstract}
Opportunistic infections are common in human immunodeficiency virus (HIV)-infected patients. Co-infections with Cryptococcus neoformans together with Mycobacterium and Pneumocystis jiroveci pneumonia (PCP) are rare, and typically occur in immunocompromised individuals, particularly AIDS patients.
\end{abstract}

\section{Introduction}

The human immunodeficiency virus (HIV) and acquired immunodeficiency syndrome (AIDS) pandemic has become a well-known global health problem specially in the last decade. Due to the effectiveness of highly active antiretroviral therapy (HAART), the incidence of new HIV infections has declined, and the number of AIDS-related deaths in adults and children worldwide has plateaued (1).

Many of the clinical features of HIV/AIDS can be ascribed to the profound immune deficiency which develops in infected patients. The destruction of the immune system by the virus results in opportunistic infection, as well as an increased risk of autoimmune disease and malignancy. HIV-related complications are rarely encountered in patients with preserved immunity (i.e. CD4 T-cell counts greater than 500 cells $/ \mathrm{mm} 3$ ). The risk of developing opportunistic infections and malignancies typical of AIDS increases progressively as CD4 counts fall below 200 cells/mm3 (2).

However, the respiratory system is still one of the most frequently affected organ systems in HIV-infected patients, and opportunistic pulmonary infections (OPIs) remain a major threat (3). The pathogens may be bacteria, mycobacteria, fungi, or viruses as well.

\section{Case presentation}

51 years old gentlemen with no previous medical history, presented with twenty days history of productive cough of yellowish sputum but sometimes mixed with minimal blood streaks. He also reported nocturnal fever, progressive shortness of breath accompanied with generalized fatigability, and significant unintentional weight loos about $15 \mathrm{Kg}$, with loss of appetite. He denies any other symptoms. He denies any history of IV drug use, tattoos. Works as a construction worker, married but he admitted to have a few extra-marital sexual relationships.

On admission he was febrile with temperature 38.9 but other vitals were within normal limits. On examination patient was cachectic and pale. neck examination was unremarkable. Chest examinations showed reduced air entery on right lower lung zone with coarse crackles and dullness percussion note. The rest of physical examination were completely unremarkable. 
His lab on admission showed bicytopenia (anemia and thrombocytopenia) with $\mathrm{Hb} 8.5 \mathrm{mg} / \mathrm{dL}$ and platelets $127 \times 10^{\wedge} 3 / \mathrm{uL}$. later on, he developed pancytopenia with WBC reaching $1.9 \times 10^{\wedge} 3 / \mathrm{uL}$ (absolute neutrophilic count $1.5 \times 10^{\wedge} 3 / \mathrm{uL}$ and lymphopenia $0.3 \times 10^{\wedge} 3 / \mathrm{uL}$ ). liver function test, renal function test, were both normal. Peripheral smear showed normocytic normochromic anemia with thrombocytopenia and lymphopenia with few reactive lymphocytes. Blood test for HIV came back positive and accordingly other tests were sent. Sputum smear for Acid fast bacilli, and PCR came negative for TB but two weeks later his sputum culture came positive for mycobacterium other than tuberculosis, and at the same time his Quantiferon test was indeterminate. He underwent bronchoscopy with bronchoalveolar lavage (BAL) and two weeks later the BAL culture result came positive for mycobacterium other than TB, and pneumocystis jiroveci was detected, as well as candida albicans. Both CMV and EBV PCR were positive from BAL. Furthermore, two bottles of blood culture showed cryptococcus neoformans growth. His rapid plasma reagin (RPR) screening for syphilis was positive, confirmatory test with Treponema pallidum antibodies was reactive as well. Urine test for chlamydia trachomatis and Neisseria gonorrhoeae DNA both were negative.

Chest x-ray showed small patchy areas of airspace shadowing in the right lung base and to a lesser extent in the right infra clavicular region (Figure A). Computed tomography scan (CT) of the neck showed bilateral cervical and supraclavicular enhancing lymph nodes, some of which demonstrate central non-enhancing areas likely representing necrosis. The largest lymph nodes are seen at level $2 \mathrm{~A}$ bilaterally measuring $8 \mathrm{~mm}$ in short axis dimension (Figure D). CT chest showed right lung ground glass nodular infiltration at posterior segment of upper lobe and apical segment of lower lobe, postero-basal collapsed consolidation. The left lung shows few nodular opacity $6 \times 4 \mathrm{~mm}$ at lung apex, postero-basal atelectatic changes. mediastinal lymph nodes are noted largest at preaortic space measures 15x11.5 mm (Figure B and C). Infectious disease department (ID) and center of communicable disease (CDC) were notified according to our hospital policy and the Patient started on appropriate treatment including trimethoprime-sulfamethoxazole and steroid. The patient was discharge after the screening of other sexual transmitted disease and CD4 count were send and to follow the result at the clinic, and then to start anti-retroviral therapy (ART) accordingly. Unfortunately, later on after several days his blood cultures turn to be positive for cryptococcus neoformans, at that time the patient went back to his home country. Two weeks later his sputum and BAL cultures came positive for mycobacterium other than tuberculosis.

\section{Discussion}

Opportunistic infections caused by bacteria and fungi are common in human immunodeficiency virus (HIV)infected patients. Cryptococcosis is one of the fungal diseases caused by Cryptococcus neoformans with higher prevalence in immunocompromised patients, especially in those with advanced HIV infection and CD4 T lymphocyte cell (CD4) counts lower than 100 cells/mm3, for whom fungaemia has been associated with a poor prognosis (4)(5). Many other infections have been characterized as opportunistic infections secondary to HIV infection, these include, but are not limited to, infections with Toxoplasma gondii, Pneumocystis jiroveci pneumonia (PCP), Cytomegalovirus (CMV), and Mycobacterium avium complex (MAC).

Furthermore, Pneumocystis jiroveci pneumonia (PCP) is major AIDS-related opportunistic infection, particularly in patients with advanced immunosuppression (CD4 count $<200 / \mu \mathrm{L}$ ) in whom human immunodeficiency virus (HIV) infection remains undiagnosed or untreated (6).

Similarly, Non-tuberculous mycobacteria (NTM) are also important causes of pulmonary and extrapulmonary disease in immunosuppressed hosts (7)(8). Distinguishing it from other opportunistic infections that occurred earlier in the course of HIV infection, for example disseminated Mycobacterium avium complex (MAC) was associated with very low CD4+ counts, generally below 50 cells $/ \mathrm{mm}^{3}(9)$.

Simultaneous infections with Cryptococcus neoformans together with Mycobacterium as well as Pneumocystis jiroveci pneumonia (PCP) are rare, and typically occur in immunocompromised individuals, particularly AIDS patients when the CD4 lymphocyte count found to be as low as 3-20/microliters, mainly if co-infection with Cryptococcus neoformans and Mycobacterium exist together (10).

In our case the patient newly found to have HIV with CD4 lymphocyte count was not sent as the patient 
loss the follow and travel back to his home country but he was found to have high viral load ( $>6$ million ), which indicate severe disease, and can explain the presence of Cryptococcus neoformans fungaemia, which it's self very rare to be found alone. At the same time, it can explain the co-infection by Cryptococcus neoformans and Non-tuberculous mycobacteria (NTM) which is also infrequent in HIV infected patients. Not only that, but existence of third infection in form of Pneumocystis jiroveci pneumonia (PCP) make our case is extremely rare. in addition, of positive PCR from BAL for Both CMV and EBV, and reactive Treponema pallidum antibodies, make the presence of other opportunistic and sexual transmitted infections is more likely in our patient.

\section{Conclusion}

Cryptococcus neoformans fungemia is not that common in HIV-infected patients, and co-infections with more than three opportunistic infections are extremely rare specially, Cryptococcus neoformans, Mycobacterium and Pneumocystis jiroveci pneumonia (PCP). for that reason, in late HIV detected cases and in advanced stages of immunosuppression, a high level of suspicion of systemic mycoses and concurrent infection by several opportunistic pathogens is required in severely immunocompromised patients.

\section{Acknowledgment}

The authors would like to acknowledge the internal medicine residency program and the Qatar National Library for their support.

\section{Statement of Ethics}

The patient consented to the publication of his case.

\section{Disclosure statement}

The authors have no conflict of interest.

\section{Funding sources}

Open Access funding provided by the Qatar National Library.

\section{Authors contributions}

Dr. Eihab A. Subahi and Fatima A. Rasoul wrote and edited the manuscript. Dr. Mohamad S. Aljafar and Haidar H. Barjas were responsible for literature review. Dr. Mohamed Abdelrazek provided us with labelled radiological images.

\section{Data availability statement}

The data that support the findings of this study are available from the corresponding author upon reasonable request.

\section{References}

1. Chou SHS, Prabhu SJ, Crothers K, Stern EJ, Godwin JD, Pipavath SN. Thoracic diseases associated with HIV infection in the era of antiretroviral therapy: Clinical and imaging findings. Radiographics . 2014 ;34(4):895-911.

2. Lloyd A. HIV infection and AIDS - PubMed. P N G Med J . 1996;39(3):174-80.

3. Benito N, Moreno A, Miro JM, Torres A. Pulmonary infections in HIV-infected patients: An update in the 21st century . Vol. 39, European Respiratory Journal. European Respiratory Society; 2012 ;39(3): $730-45$.

4. Jonathan E Kaplan, Constance Benson, King K Holmes, John T Brooks, Alice Pau HM. Guidelines for Prevention and Treatment of Opportunistic Infections in HIV-Infected Adults and Adolescents Recommendations from CDC, the National Institutes of Health, and the HIV Medicine Association of the Infectious Diseases Society of America. MMWR Recomm Rep. 2009;58(RR-4):1-207. 
5. Witt MD, Lewis RJ, Larsen RA, Milefchik EN, Leal MAE, Haubrich RH, et al. Identification of patients with acute AIDS-associated cryptococcal meningitis who can be effectively treated with fluconazole: The role of antifungal susceptibility testing. Clin Infect Dis. 1996;22(2):322-8.

6. Shibata S, Kikuchi T. Pneumocystis pneumonia in HIV-1-infected patients Vol. 57, Respiratory Investigation. Elsevier B.V.; 2019 ;57(3): 213-219.

7. Marino A, Caltabiano E, Zagami A, Onorante A, Zappalà C, Locatelli ME, et al. Rapid emergence of cryptococcal fungemia, Mycobacterium chelonae vertebral osteomyelitis and gastro intestinal stromal tumor in a young HIV late presenter: a case report. BMC Infect Dis. 2018 Dec 27;18(1):693.

8. Henkle E, Winthrop KL. Nontuberculous mycobacteria infections in immunosuppressed hosts. Vol. 36, Clinics in Chest Medicine. W.B. Saunders; 2015;36(1):91-99.

9. Havlik JA, Horsburgh CR, Metchock B, Williams PP, Fann SA, Thompson SE. Disseminated Mycobacterium avium Complex Infection: Clinical Identification and Epidemiologic Trends. J Infect Dis. 1992 Mar 1;165(3):577-80.

10. Arastéh K, Cordes C, Futh U, Grosse G, Dietz E, Staib F. Co-infection by cryptococcus neoformans and mycobacterium avium intracellulare in AIDS: Clinical and epidemiological aspects. Mycopathologia. 1997;140(3):115-20.

\section{List of figures}

\begin{tabular}{ll}
\hline figure & Description \\
\hline A & Chest X-ray PA view shows small patchy areas of airspace shadowing in the right lung base and to a lesser extent \\
B & Axial CT scan of the chest lung window show small patchy areas of ground glass and nodular infiltration in the po \\
C & Axial CT scan of the chest lung window show small patchy areas of ground glass and nodular infiltration in the Ap \\
D & Axial post contrast study of the neck shows bilateral cervical sub centimetric enhancing lymph nodes, some of then \\
\hline
\end{tabular}




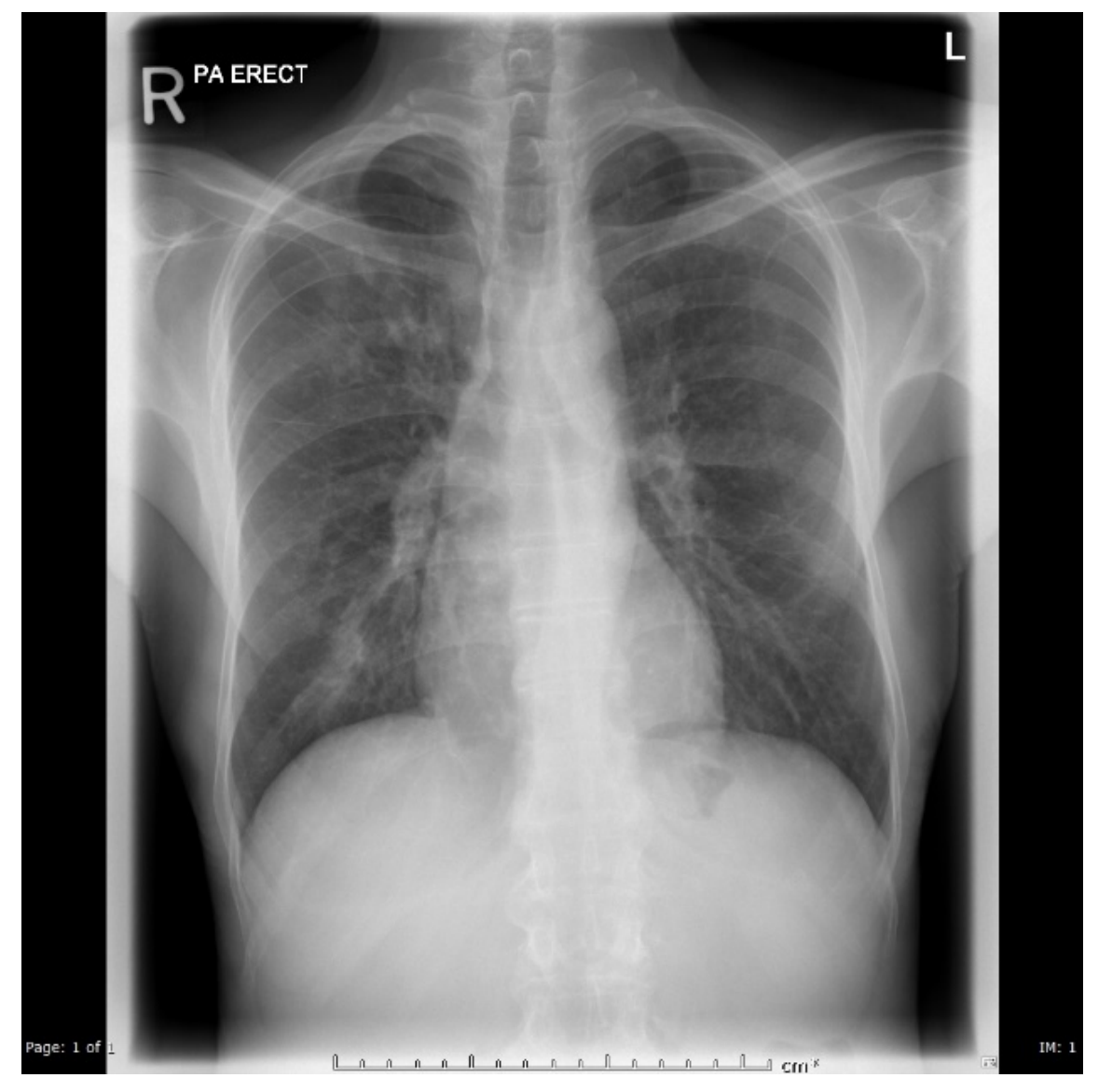

A 


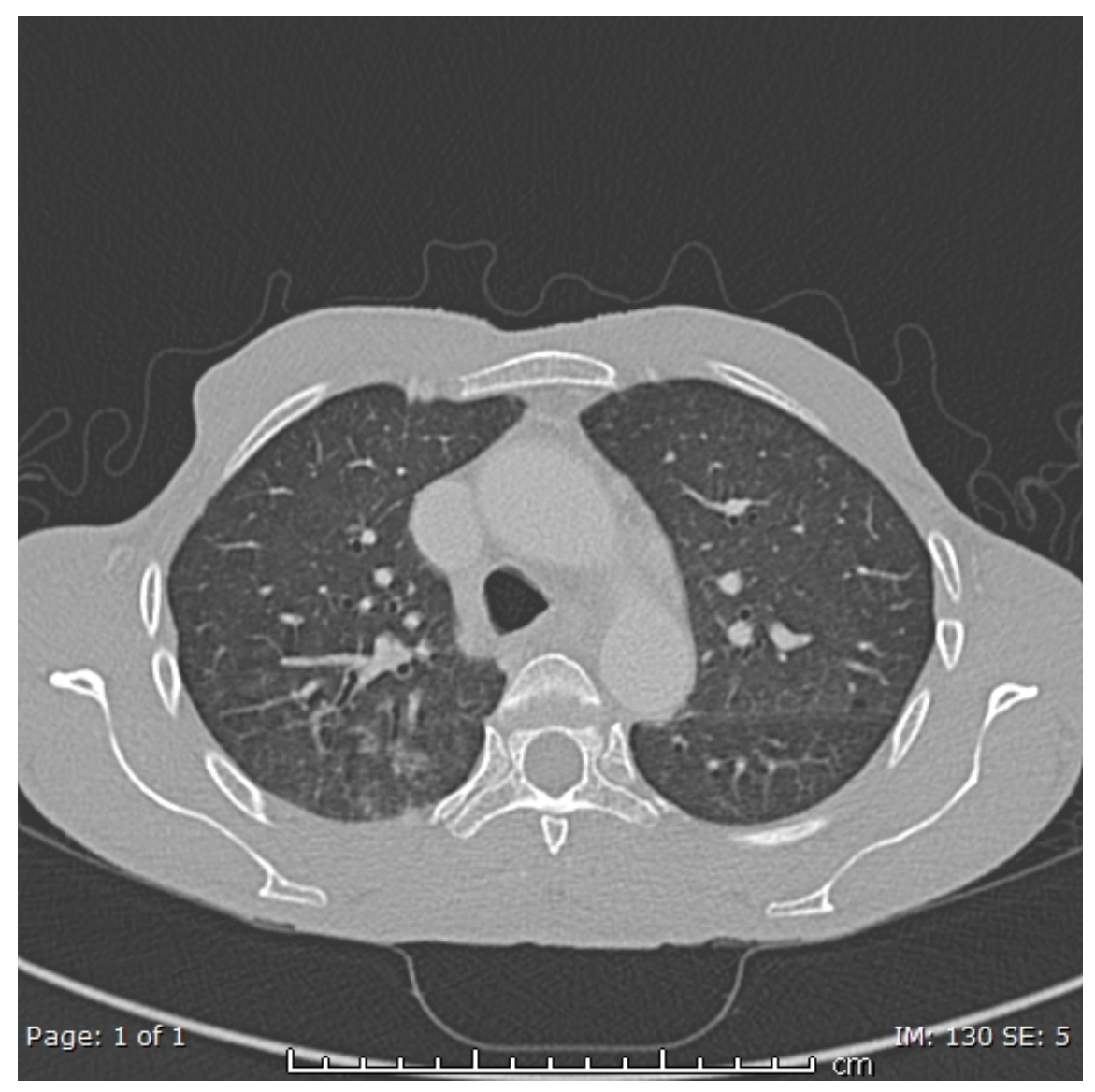




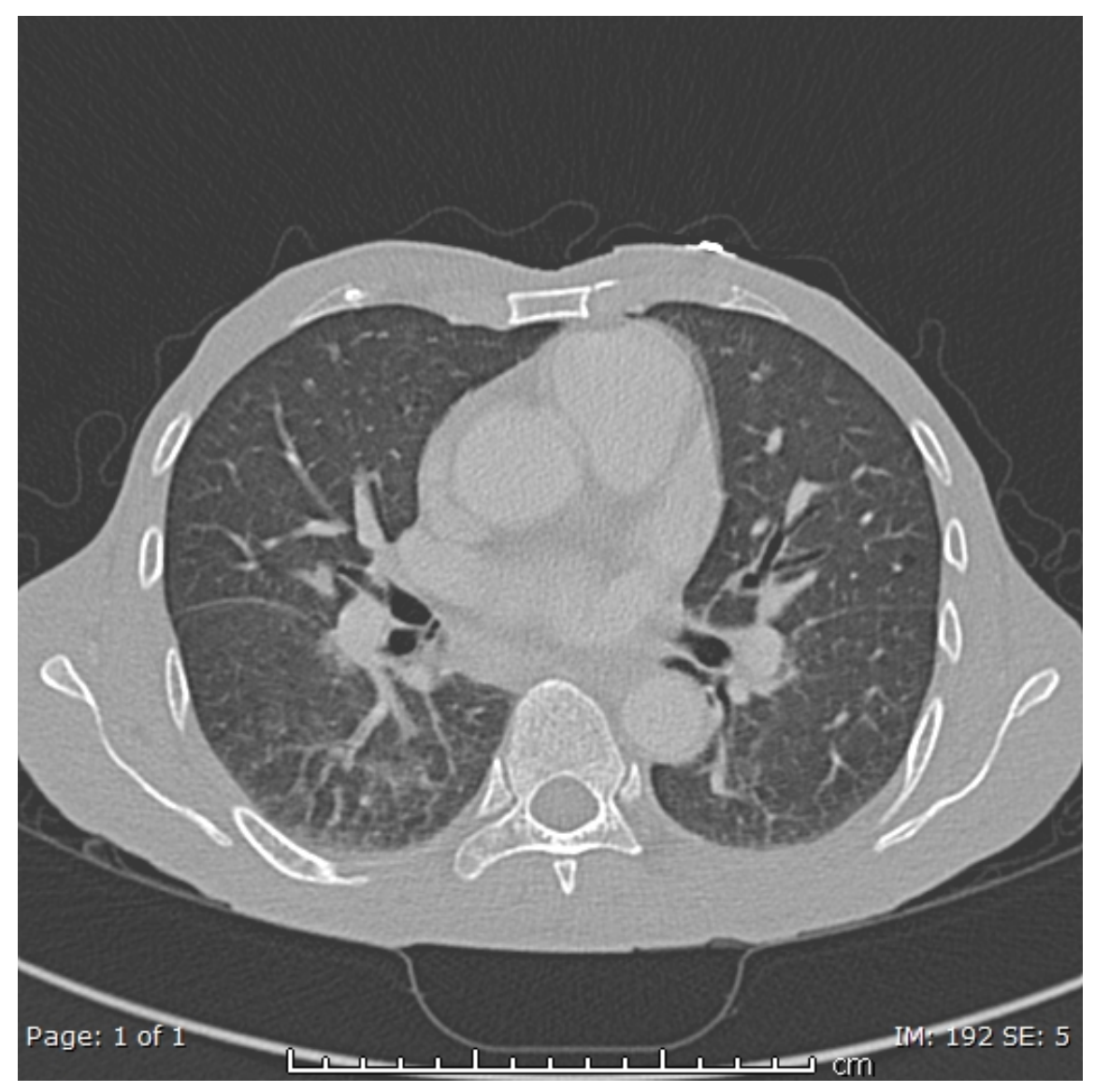

C 


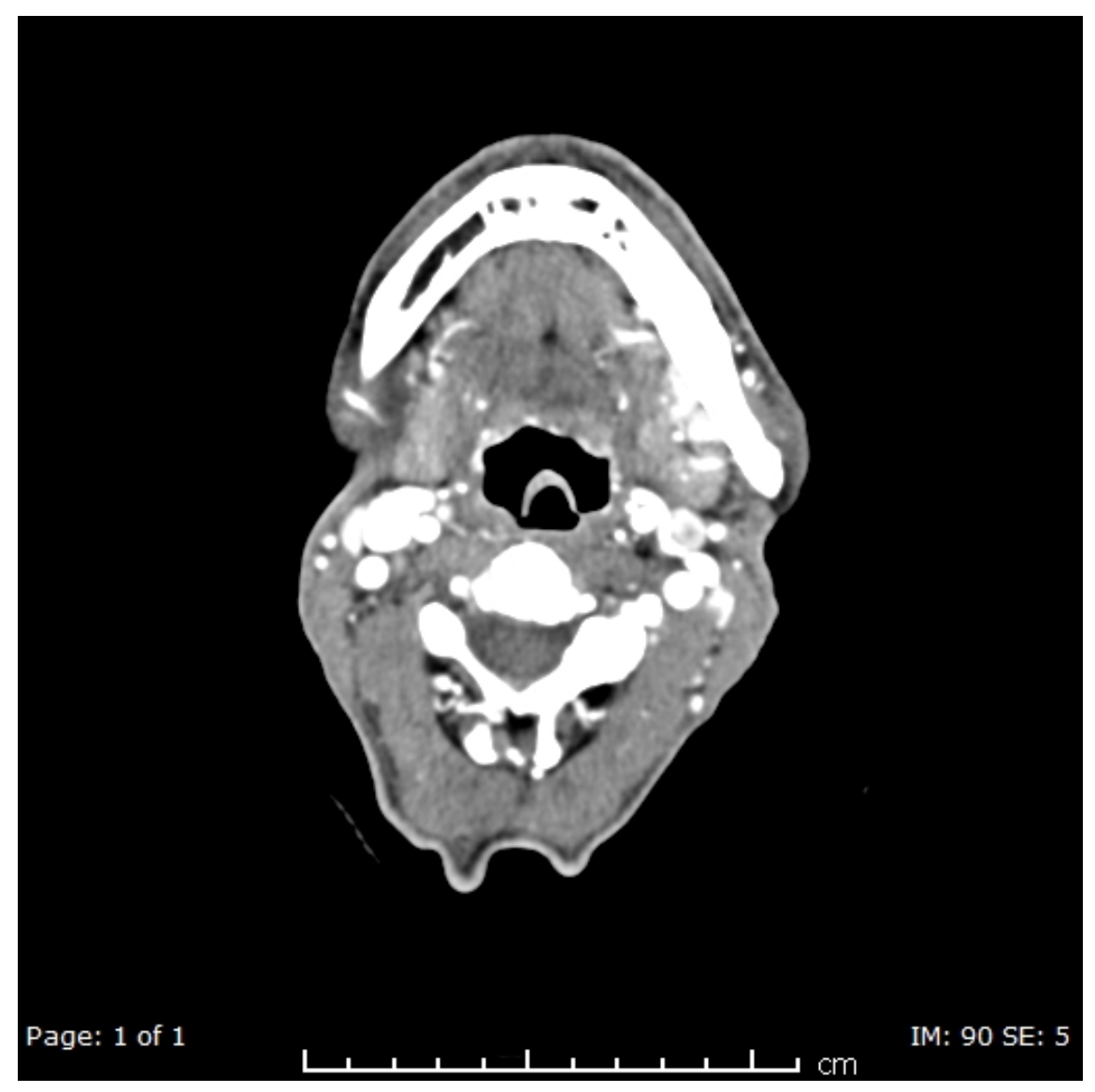

$\mathrm{D}$

Chest X-ray PA view shows small patchy areas of airspace shadowing in the right lung base and to a lesser extent in the right infra clavicular region (Arrows).

B \&C) Axial CT scan of the chest lung window show small patchy areas of ground glass and nodular infiltration in the posterior segment of the right lung upper lobe (arrow in B) and in the Apical segment of the right lower lobe (Arrow in C). D) Axial post contrast study of the neck shows bilateral cervical sub centimetric enhancing lymph nodes, some of them show central non-enhancing areas likely representing necrosis (Arrow) 